\title{
Tetra-n-butylammoniumdicyanoaurat $(\mathrm{I}),\left(n-\mathrm{C}_{4} \mathrm{H}_{9}\right)_{4} \mathrm{NAu}(\mathrm{CN})_{2}$
}

Tetra- $n$-butylammonium Dicyanoaurate(I), $\left(n-\mathrm{C}_{4} \mathrm{H}_{9}\right)_{4} \mathrm{NAu}(\mathrm{CN})_{2}$

R. J. Schubert und K.-J. Range*

Institut für Anorganische Chemie der Universität Regensburg,

Universitätsstraße 31, D-8400 Regensburg

Herrn Prof. Dr. D. Babel zum 60. Geburtstag gewidmet

Z. Naturforsch. 45b, 1118-1122 (1990); eingegangen am 12. Februar 1990

Tetra- $n$-butylammonium Dicyanoaurate(I), Crystal Growth, Crystal Structure

Single crystals of $\left(n-\mathrm{C}_{4} \mathrm{H}_{9}\right)_{4} \mathrm{NAu}(\mathrm{CN})_{2}$ were obtained by crystallization using the temperature decreasing method in a newly developed computer-controlled apparatus. The colourless crystals are monoclinic, space group A2/n, with $a=20.216(2) \AA, b=13.130(1) \AA, c=$ $9.328(1) \AA$, and $\beta=109.69(1)^{\circ}$. The measured density is $1.45 \mathrm{~g} / \mathrm{cm}^{3}$, which agrees with the calculated density of $1.40 \mathrm{~g} / \mathrm{cm}^{3}$ for $Z=4$. The structure was solved by Direct Methods using 674 independent reflections and refined by least-squares procedures to conventional and weighted $R$ factors of 0.068 and 0.058 , respectively. The nearly linear $\left[\mathrm{Au}(\mathrm{CN})_{2}\right]^{-}$-anions form pseudohexagonal layers, in which they are arranged parallel to each other. The shortest $\mathrm{Au}-\mathrm{Au}$ distance is $8.05 \AA$. The stacking of the layers produces pseudo-trigonal channels, occupied by the cations. The tetra- $n$-butylammonium cation has four ordered trans chains with idealized $\mathrm{D}_{2 \mathrm{~d}}$ symmetry $(\overline{4} 2 \mathrm{~m})$.

Im Rahmen unserer Untersuchungen über den Einfluß unterschiedlich großer Kationen auf die Kristallstrukturen von Verbindungen mit annähernd linearen, stäbchenförmigen Anionen beschäftigen wir uns mit Dicyanoargentaten und -auraten. Nachdem wir eine Reihe von Kristallstrukturen mit Alkali- und Erdalkaliionen als Kationen aufklären konnten [1-4], waren für uns auch Verbindungen mit noch größeren Kationen interessant. Im folgenden berichten wir über die Darstellung, Kristallisation und Kristallstruktur von Tetra- $n$-butylammoniumdicyanoaurat(I).

Mason [5] teilte in einer Arbeit über ChargeTransfer-Effekte in $\left(n-\mathrm{C}_{4} \mathrm{H}_{9}\right)_{4} \mathrm{NAu}(\mathrm{CN})_{2}$ unvollständige Kristalldaten für die Verbindung mit. Einzelheiten hierzu wurden jedoch nicht publiziert [6].

\section{Experimentelles}

Durch Zusammengeben äquimolarer Lösungen von $\left(n-\mathrm{C}_{4} \mathrm{H}_{9}\right)_{4} \mathrm{NCl}$ und $\mathrm{KAu}(\mathrm{CN})_{2}$ erhält man einen weißen, mikrokristallinen Niederschlag von Tetra- $n$-butylammoniumdicyanoaurat. Dieser wird abgenutscht und mit Eiswasser gewaschen. Das Produkt wird in Wasser oder Ethanol/Wasser (1:2) umkristallisiert. Anschließend wird über $\mathrm{P}_{4} \mathrm{O}_{10}$ im Vakuum getrocknet. Die Ausbeute liegt bei

\footnotetext{
* Sonderdruckanforderungen an Prof. Dr. K.-J. Range. Verlag der Zeitschrift für Naturforschung, D-7400 Tübingen 0932-0776/90/0800-1118/\$01.00/0
}

$80 \%$. Anschließende Guinieraufnahmen zeigten keine Reflexe von $\mathrm{KAu}(\mathrm{CN})_{2}$ oder $\left(n-\mathrm{C}_{4} \mathrm{H}_{9}\right)_{4} \mathrm{NCl}$ im Produkt. Die Schmelzpunktbestimmung ergab einen Schmelzbereich von 65-69 ${ }^{\circ}$ C. DSC-Messungen $\left(10 \mathrm{~K} \mathrm{~min}^{-1}\right)$ lieferten einen Schmelzpeak bei $73{ }^{\circ} \mathrm{C}$.

Elementaranalyse [\%]

Ber. Au 40,08 C 43,99 H 7,38 N 8,55,

Gef. Au 38,76 C 42,78 H 7,42 N 8,21.

Um für Einkristalluntersuchungen geeignete Kristalle zu erhalten, wurde die Kristallisationsmethode nach dem Prinzip der Temperaturabsenkung gesättigter Lösungen angewandt. Mit Hilfe einer neu entwickelten, computergesteuerten Kristallisationsanlage erhielten wir klare Einkristalle von $c a .200 \times 300 \times 300 \mu \mathrm{m}$ Größe. Das steuerbare Verfahren liefert auch dann noch brauchbare Einkristalle, wenn mit der sonst üblichen Verdunstungsmethode keine ausreichend guten Kristallisationsergebnisse zu erzielen sind. Die bestehende Konfiguration erlaubt bei einer Temperaturkonstanz von $\pm 0,01{ }^{\circ} \mathrm{C}$ und einem Arbeitsbereich von $10-80^{\circ} \mathrm{C}$ Abkühlzeiten von mehreren Monaten. Aufbau und Funktionsweise sind an anderer Stelle ausführlich beschrieben [7].

\section{Strukturanalyse}

Für die Strukturanalyse wurden geeignete Kristalle mit ca. $130 \times 290 \times 300 \mu \mathrm{m}$ Größe ausgewählt. Weissenbergaufnahmen ergaben die Laueklasse 2/m. Unter Berücksichtigung der Donnay- 
Konvention $c<a$ verwiesen integrale Auslöschungen $(h k l: k+\ell \neq 2 n)$ auf eine A-Zentrierung, zonale Auslöschungen $(h 0 l: \ell+h \neq 2 n)$ führten zu den monoklinen Raumgruppen A $1 n 1$ bzw. A 1 2/n 1 (Nicht-Standard-Aufstellung von Cc, Nr. 9, bzw. C $2 / c$, Nr. 15). Die Sammlung der Reflexintensitäten erfolgte an einem Enraf-NoniusCAD 4-Diffraktometer (Graphitmonochromator, MoK $a$-Strahlung, $\lambda=0,71073 \AA$, $\mathrm{T}=25^{\circ} \mathrm{C}$ ). Im Bereich $2^{\circ}<\theta<20^{\circ}$ wurden 3064 Reflexe in $\omega-2 \theta$-scan registriert. Drei in regelmäßigen $\mathrm{Ab}$ ständen gemessene Referenzreflexe zeigten einen deutlichen Zerfall des Kristalls an, der Intensitätsverlust während der Messung betrug 27\%. Weitere Einzelheiten zur Datensammlung finden sich in Tab. I.

Ein N(Z)-Test machte das Vorliegen der zentrosymmetrischen Raumgruppe A2/n wahrscheinlich. Das Ergebnis der Strukturrechnung bestätigte diese Annahme. Nach der Datenreduktion verblieben 674 symmetrieunabhängige Reflexe mit $\mathrm{F}>3 \sigma(\mathrm{F})$. Die Lage der Edelmetallatome konnte mit Direkten Methoden (Programm SHELXS-86 [8]) gefunden werden. Anschließende Fourierund Differenz-Fourier-Synthesen (Programm
SHELX-76 [9]) zeigten die C- und N-Atome. Absorptionseffekte wurden numerisch korrigiert (Programm DIFABS [10]). Least-Squares-Verfeinerungen der Ortsparameter und Temperaturfaktoren (anisotrop für $\mathrm{Au}$, isotrop für $\mathrm{C}$ und $\mathrm{N}$ ) konvergierten bei $R=0,068$ bzw. $R_{\mathrm{w}}=0,058$. $\mathrm{H}$-Atome wurden nicht berücksichtigt.

Nach Kenntnis der Einkristalldaten konnte das entsprechende Guinierdiagramm (Huber-GuinierSystem 600, Quarzmonochromator, $\mathrm{CuKa} a_{1}$, Ge als Standard) vollständig indiziert werden. Die in Tab. I aufgeführten Gitterkonstanten wurden durch eine Least-Squares-Verfeinerung der Guinierdaten erhalten. Ortsparameter, Temperaturfaktoren, wichtige Atomabstände und Bindungswinkel sind in Tab. II und III zusammengestellt*.

\footnotetext{
* Weitere Einzelheiten zur Kristallstrukturuntersuchung können beim Fachinformationszentrum Karlsruhe, Gesellschaft für wissenschaftlich-technische Information mbH, D-7514 Eggenstein-Leopoldshafen 2, unter Angabe der Hinterlegungsnummer CSD 54653, des Autors und des Zeitschriftenzitats angefordert werden.
}

\begin{tabular}{|c|c|}
\hline Raumgruppe & A $12 / n 1(\mathrm{Nr} .15)$ \\
\hline \multicolumn{2}{|l|}{ Gitterkonstanten* } \\
\hline$a[\AA]$ & $20,216(2)$ \\
\hline$b[\AA]$ & $13,130(1)$ \\
\hline$c[\AA]$ & $9,328(1)$ \\
\hline$\beta\left[{ }^{\circ}\right]$ & $109,69(1)$ \\
\hline Volumen der EZ $\left[\AA^{3}\right]$ & 2331 \\
\hline Formeleinheiten/EZ & 4 \\
\hline Dichte $_{\exp .}\left[\mathrm{g} \cdot \mathrm{cm}^{-3}\right]$ & 1,45 \\
\hline Dichte $_{\text {rönt }}\left[\mathrm{g} \cdot \mathrm{cm}^{-3}\right]$ & 1,40 \\
\hline Kristallgröße $[\mu \mathrm{m}]$ & $130 \times 290 \times 300$ \\
\hline Diffraktometer & Enraf-Nonius CAD 4 \\
\hline Strahlung, Monochromator & $\operatorname{MoK} \alpha(\lambda=0,71073 \AA)$, Graphit \\
\hline Meßgeometrie & $\omega / 2 \theta$-scan \\
\hline Abtastwinkel [ $]$ & $(1,0+0,34 \tan \theta)$ \\
\hline$(\sin \theta / \lambda)_{\max }\left[\AA^{-1}\right]$ & 0,481 \\
\hline$h k l$-Grenzen & $-9<h<7,-12<k<13,-10<\ell<20$ \\
\hline Intensitätsverlust & $-27,1 \%$ \\
\hline Gemessene Reflexe & 3064 \\
\hline Beobachtete Reflexe $[\mathrm{I}>1 \sigma(\mathrm{I})]$ & 1147 \\
\hline Unabhängige Reflexe $[\mathrm{F}>3 \sigma(\mathrm{F})], \mathrm{R}_{\text {int }}$ & $674,0,054$ \\
\hline Absorptionskoeffizient $\left[\mathrm{mm}^{-1}\right]$ & 6,56 \\
\hline Absorptionskorrektur & DIFABS \\
\hline Variable, $(\Delta / \sigma)_{\max }$ & $49,0,01$ \\
\hline$R, R_{\mathrm{w}}$ & $0,068,0,058$ \\
\hline 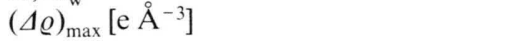 & $+1,62,-1,18$ \\
\hline
\end{tabular}

Tab. I. Kristallographische Daten und Daten zur Strukturanalyse von $\left(n-\mathrm{C}_{4} \mathrm{H}_{9}\right)_{4} \mathrm{Au}(\mathrm{CN})_{2}$. (Standardabweichungen der letzten Stelle sind wie in allen Tabellen in Klammern angegeben.)

\footnotetext{
* Die angegebenen Gitterkonstanten wurden durch Least-Squares-Verfeinerung von Guinierdaten erhalten.
} 
Tab. II. Ortsparameter und isotrope Temperaturfaktoren $\left[\AA^{2}\right]$ von $\left(n-\mathrm{C}_{4} \mathrm{H}_{9}\right)_{4} \mathrm{NAu}(\mathrm{CN})_{2}$.

\begin{tabular}{lllll}
\hline Atom & $x / a$ & $y / b$ & $z / c$ & $\mathrm{U}_{\text {eq }} / \mathrm{U}_{\text {iso }}$ \\
\hline Au & 0 & $3 / 4$ & $1 / 4$ & $0,100(1)^{*}$ \\
C(CN) & $0,088(2)$ & $0,716(2)$ & $0,413(4)$ & $0,14(2)$ \\
N(CN) & $0,136(2)$ & $0,706(2)$ & $0,496(3)$ & $0,17(2)$ \\
N(Amm) & $1 / 4$ & $0,951(2)$ & $1 / 4$ & $0,09(1)$ \\
C11 & $0,194(1)$ & $0,877(2)$ & $0,146(3)$ & $0,070(8)$ \\
C12 & $0,131(2)$ & $0,937(2)$ & $0,031(3)$ & $0,078(9)$ \\
C13 & $0,084(2)$ & $0,852(2)$ & $0,930(3)$ & $0,12(1)$ \\
C14 & $0,016(2)$ & $0,897(2)$ & $0,834(3)$ & $0,16(5)$ \\
C21 & $0,217(2)$ & $0,024(2)$ & $0,342(3)$ & $0,085(9)$ \\
C22 & $0,181(2)$ & $0,966(2)$ & $0,433(3)$ & $0,11(1)$ \\
C23 & $0,163(2)$ & $0,064(2)$ & $0,528(3)$ & $0,19(2)$ \\
C24 & $0,117(2)$ & $0,013(3)$ & $0,601(5)$ & $0,22(2)$ \\
\hline
\end{tabular}

* $U_{\text {eq }}$ für Au ist definiert als ein Drittel der Spur des orthogonalisierten $\mathrm{U}_{\mathrm{ij}}$-Tensors.

\section{Diskussion}

Jedes Goldatom bildet mit zwei Cyanogruppen ein nahezu lineares Anion $\left(\mathrm{C}-\mathrm{Au}-\mathrm{C} 180^{\circ}\right.$, $\left.\mathrm{Au}-\mathrm{C}-\mathrm{N} 174(3)^{\circ}\right)$. Die $\left[\mathrm{Au}(\mathrm{CN})_{2}\right]^{-}$-Hanteln bilden pseudohexagonale Schichten (Abb. 1), in denen sie parallel zueinander angeordnet sind. In den so entstehenden pseudotrigonalen Kanälen längs der $a$-Achse (Abb. 2) finden zwischen den Goldschichten die Tetra- $n$-butylammonium-Kationen Platz (Abb. 3).

Der kürzeste $\mathrm{Au}-\mathrm{Au}$-Abstand beträgt $8,05 \AA$ [9]. Er ist damit erheblich größer als die $\mathrm{Au}-\mathrm{Au}-$ Abstände in Alkalidicyanoauraten $(3,1-3,7 \AA$ ) [4, 11-13]. Die Ladungsschwerpunkte von Kation und Anion sind 5,70(1) $\AA$ voneinander entfernt.

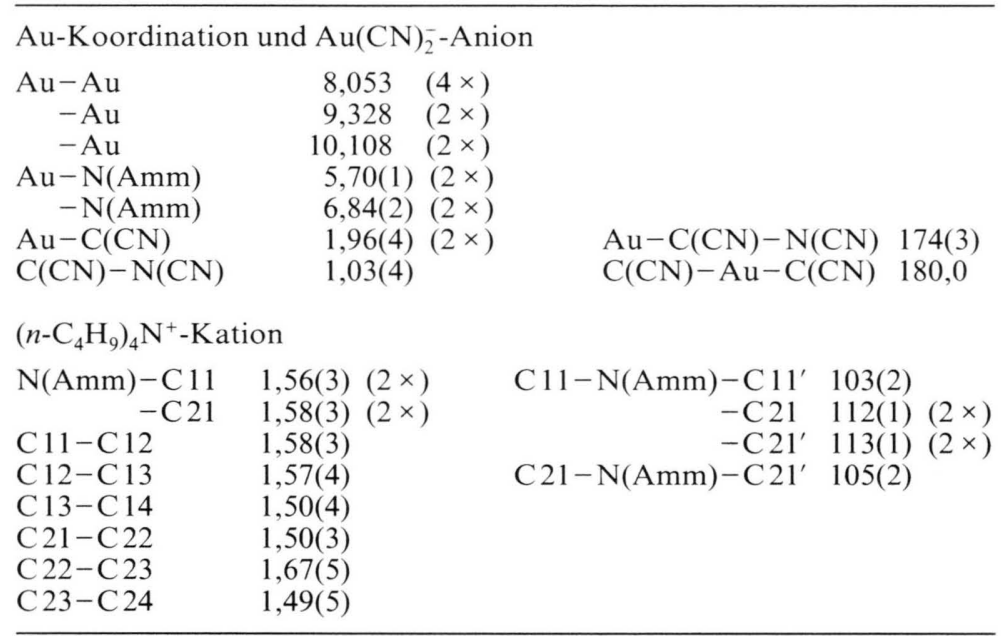

Tab. III. Wichtige Atomabstände [Å] und Winkel $\left[{ }^{\circ}\right]$ in $\left(n-\mathrm{C}_{4} \mathrm{H}_{9}\right)_{4} \mathrm{NAu}(\mathrm{CN})_{2}$.

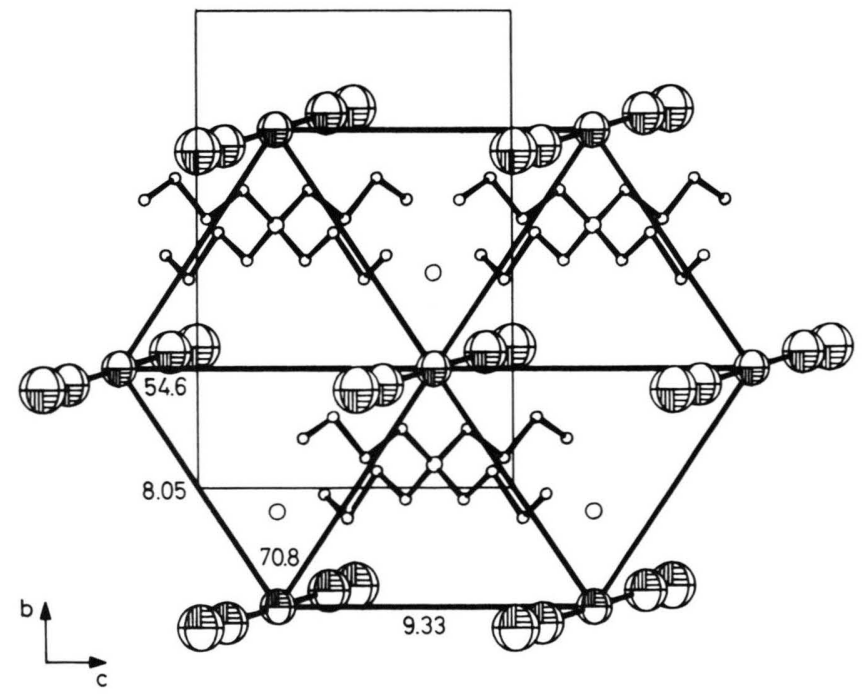

Abb. 1. Pseudohexagonale Schicht aus $\left[\mathrm{Au}(\mathrm{CN})_{2}\right]^{-}$-Hanteln. Die Kationen unter der Schicht sind der Übersicht halber nur angedeutet. 


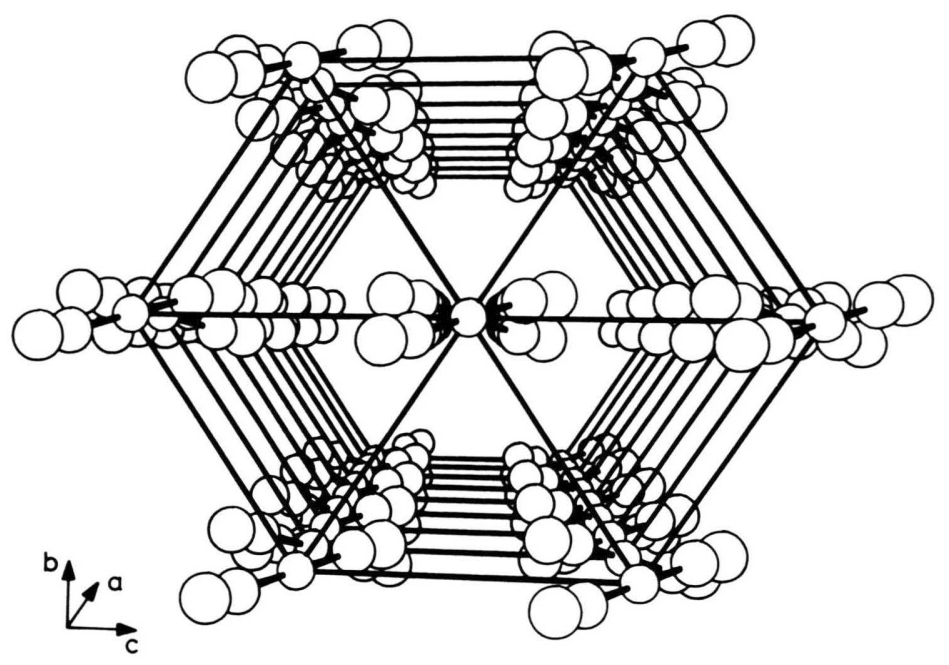

Abb. 2. Pseudotrigonale Kanäle entlang der $a$-Achse.
Der Zusammenhalt des Kristallgitters sollte daher wesentlich durch van-der-Waals-Kräfte bedingt sein.

Wie Abb. 4 zeigt, bildet das Tetra- $n$-butylammonium-Kation in $\left(n-\mathrm{C}_{4} \mathrm{H}_{9}\right)_{4} \mathrm{NAu}(\mathrm{CN})_{2}$ keine kugelförmige Konformation aus, sondern ein planares Kreuz mit $\mathrm{D}_{2 \mathrm{~d}}$-Symmetrie $(\overline{4} 2 \mathrm{~m})$. Alle Butylketten haben trans-Konformation (4t). Denkbar wäre auch eine angenähert tetraedrische Anordnung der Butylketten um das Zentralatom Stickstoff $\left(\mathrm{S}_{4}\right.$-Symmetrie ( $\left.\left.\overline{4}\right)\right)$. Sie entsteht bei der Rotation aller Butylketten um die jeweilige $\mathrm{N}-\mathrm{C}_{\alpha}$-Bindung um $120^{\circ}$ und in gleicher Richtung. Dadurch behalten die Wasserstoffatome den maximal möglichen Abstand zueinander. Beide Konformatio- nen sollten deshalb auf ähnlichen Energieniveaus liegen [14]. Dennoch sind überwiegend Tetra- $n$ butylammonium-Verbindungen bekannt, die ein Kation mit (zumindest angenäherter) $\mathrm{D}_{2 \mathrm{~d}}$-Symmetrie besitzen [15]. In allen Tetra- $n$-butylammonium-Verbindungen treten relativ große Temperaturschwingungen im Kation auf. Sie steigen vom Zentralatom Stickstoff bis zu den Enden der Butylketten auf meist mehr als das Doppelte an. $\mathrm{U}_{\mathrm{eq}}{ }^{-}$ Werte $>0,20 \AA^{2}$ sind bekannt [16]. Dies bedeutet eine relativ freie Beweglichkeit der Butylketten, das zentrale Stickstoffatom sitzt dagegen fest, der Schwerpunkt des Kations ist also definiert.

Wegen der starken Temperaturschwingungen der Kohlenstoffatome ist es unwahrscheinlich, daß

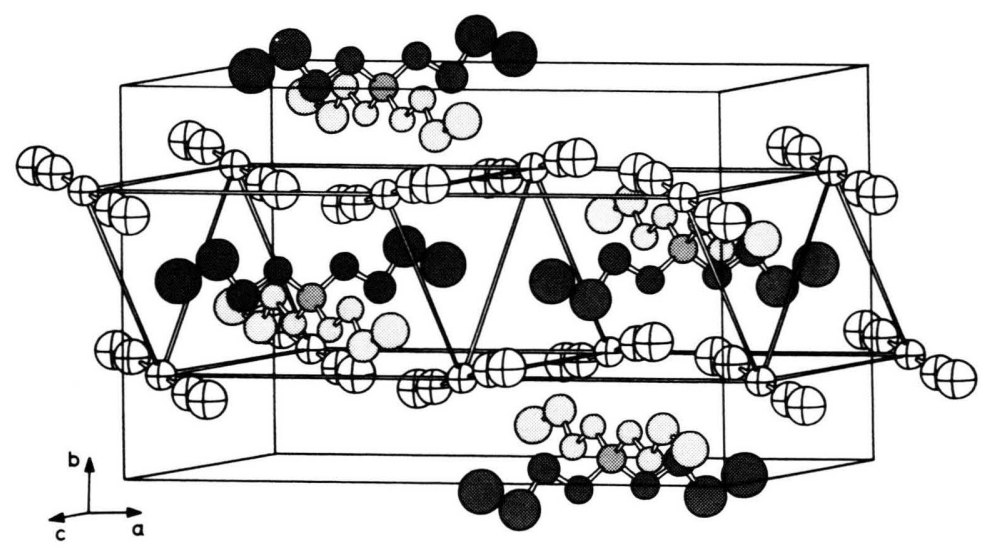

Abb. 3. Elementarzelle von $\left(n-\mathrm{C}_{4} \mathrm{H}_{9}\right)_{4} \mathrm{NAu}(\mathrm{CN})_{2}$. 

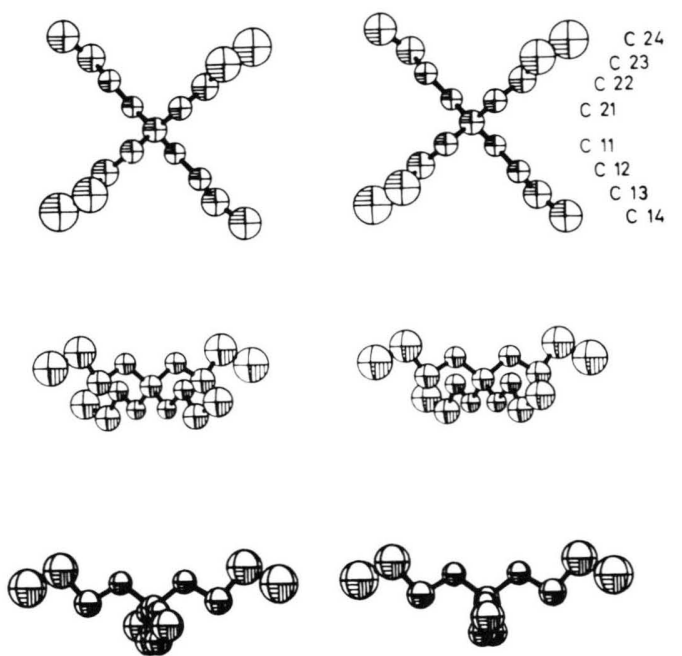

Abb. 4. Das $\left(n-\mathrm{C}_{4} \mathrm{H}_{9}\right)_{4} \mathrm{~N}^{+}$-Kation in $\mathrm{D}_{2 \mathrm{~d}}-$ Konformation. das jeweilige Anion einen Teil des Raumes beanspruchen kann, der zwischen den Butylketten verbleibt.

Eine Überschlagsrechnung ergibt, daß der Platzbedarf eines $\left(n-\mathrm{C}_{4} \mathrm{H}_{9}\right)_{4} \mathrm{~N}^{+}$-Kations mit $\mathrm{D}_{2 \mathrm{~d}^{-}}$ Symmetrie etwa um $15 \%$ geringer ist als für $\mathrm{S}_{4}$ Symmetrie. Mit entsprechend großen Anionen kann so eine dichtere Packung verwirklicht werden.

Herrn Dr. U. Klement danken wir für die Sammlung der Diffraktometerdaten. Der Fonds der Chemischen Industrie und die Degussa AG förderten unsere Arbeiten durch eine Forschungsbeihilfe.
[1] K.-J. Range, M. Zabel, H. Meyer und H. Fischer, Z. Naturforsch. 40 b, 1618 (1985).

[2] K.-J. Range, S. Kühnel und M. Zabel, Acta Crystallogr. C 45, 1419 (1989).

[3] M. Zabel, S. Kühnel und K.-J. Range, Acta Crystallogr. C 45, 1619 (1989).

[4] R. J. Schubert und K.-J. Range, Z. Naturforsch. 45b, 629 (1990).

[5] W. R. Mason, J. Am. Chem. Soc. 98, 5182 (1976).

[6] W. R. Mason, persönliche Mitteilung (1987).

[7] R. J. Schubert, Dissertation, Regensburg (1989).

[8] G. M. Sheldrick, SHELXS-86. A Program for Crystal Structure Determination, Universität Göttingen (1986).

[9] G. M. Sheldrick, SHELX-76. A Program for Crystal Structure Determination, University of Cambridge, England (1976).
[10] N. Walker und D. Stuart, Acta Crystallogr. A39, 159 (1983)

[11] A. Rosenzweig und D. T. Cromer, Acta Crystallogr. B 12, 705 (1959).

[12] N. Blom, A. Ludi, H.-B. Bürgi und K. Tichy, Acta Crystallogr. C 40, 1767 (1984).

[13] N. Blom, A. Ludi und H.-B. Bürgi, Acta Crystallogr. C40, 1770 (1984).

[14] M. R. Snow und J. A. Ibers, Inorg. Chem. 12, 249 (1973).

[15] Zusammenstellungen strukturell charakterisierter Tetra- $n$-butylammonium-Verbindungen finden sich bei Snow und Ibers [14] sowie Schubert [7].

[16] J. D. Forester, A. Zalkin und D. H. Templeton, Inorg. Chem. 3, 1507 (1964). 